\title{
Classification of Chronic Back Muscle Degeneration after Spinal Surgery and Its Relationship with Low Back Pain
}

Seiji Ohtori, Sumihisa Orita, Kazuyo Yamauchi, Yawara Eguchi, Yasuchika Aoki, Junichi Nakamura, Tetsuhiro Ishikawa, Masayuki Miyagi, Hiroto Kamoda, Miyako Suzuki, Gou Kubota, Kazuhide Inage, Takeshi Sainoh, Jun Sato, Yasuhiro Shiga, Koki Abe, Kazuki Fujimoto, Hirohito Kanamoto, Gen Inoue, Kazuhisa Takahashi

Department of Orthopaedic Surgery, Graduate School of Medicine, Chiba University, Chiba, Japan

\section{Study Design: Retrospective case series.}

Purpose: To classify back muscle degeneration using magnetic resonance imaging (MRI) and investigate its relationship with back pain after surgery.

Overview of Literature: Back muscle injury and degeneration often occurs after posterior lumbar surgery, and the degeneration may be a cause of back pain. However, the relationship between back muscle degeneration and back pain remains controversial.

Methods: A total of 84 patients (average age, 65.1 years; 38 men, 46 women) with lumbar spinal stenosis underwent posterior decompression surgery alone. MRI (1.5 tesla) was evaluated before and more than a year after surgery in all patients. Muscle on MRI was classified into three categories: low intensity in T1-weighted imaging, high intensity in T2-weighted imaging (type 1), high intensity in both T1- and T2-weighted images (type 2), and low intensity in both T1- and T2-weighted imaging (type 3). The prevalence of the types and their relationship with back pain (determined on a visual analog scale) were evaluated.

Results: MRI revealed muscle degeneration in all patients after surgery (type 1,6\%; type 2, 82\%; and type 3,12\%). Type 2 was significantly more frequent compared with types 1 and 3 ( $p<0.01)$. Low back pain was significantly improved after surgery $(p<0.01)$. Low back pain was not associated with any MRI type of muscle degeneration after surgery $(p>0.05)$.

Conclusions: Various pathologies of back muscle degeneration after posterior lumbar surgery were revealed. Type 2 (fatty) change was most frequent, and other patients had type 3 (scar) or type 1 (inflammation or water-like) changes. According to the Modic classification of bone marrow changes, Modic type 1 change is associated with inflammation and back pain. However, no particular type of back muscle degeneration was correlated with back pain after surgery.

Keywords: Lumbar; Muscle; Injury; Surgery; Pain

\section{Introduction}

In Sweden, up to $25 \%$ of patients report unimproved or worse pain and up to $40 \%$ are not happy with the outcome of lumbar fusion [1]. There are many possible reasons for poor results, including instrumentation failure,

\footnotetext{
Received Sep 26, 2015; Revised Oct 23, 2015; Accepted Oct 24, 2015

Corresponding author: Seiji Ohtori

Department of Orthopaedic Surgery, Graduate School of Medicine, Chiba University,

1-8-1 Inohana, Chuo-ku, Chiba 260-8670, Japan

Tel.: +81-43-226-2117, Fax: +81-43-226-2116, E-mail: sohtori@faculty.chiba-u.jp
} 
inadequate surgical technique, and poor patient selection $[2,3]$. Other reported reasons for poor surgical results include presurgical pain/function, negative personality traits, emotional status, anxiety/depression, negative outcome expectations, negative coping, and duration of back pain, muscle injury, and workers' compensation $[2,3]$. However, the relationship between back muscle injury after surgery and pain remains unclear.

Lumbar surgery induces histological change in muscle in humans. Kawaguchi et al. [4] reported the histological and histochemical changes in back muscle in patients who underwent surgery for lumbar spine disorders for the first time and in patients who underwent repeated posterior lumbar surgery. Histological damage to back muscle because of previous surgical intervention was long lasting. Of 178 patients who had undergone laminectomies 2-5 years earlier, 14 patients had good recovery and 21 patients had poor recovery [5]. Back muscle biopsy was performed in patients with poor outcome. Local denervation, atrophy of paraspinal muscles, and loss of muscular support leads to disability and increased biomechanical strain, and possibly failed back syndrome and pain [5].

The cross-sectional area (CSA) of the back musculature has been measured before and after various types of posterior lumbar surgery in T2-weighted axial magnetic resonance imaging (MRI) using a computer-linked digitizer [6]. The extent of atrophy of the back musculature was determined on MRI after five types of posterior lumbar surgery. However, the clinical outcomes showed no correlation with the extent of atrophy after each surgical procedure [7]. This finding suggested limited application of back muscle atrophy as an indicator of pain.

Back muscle injury and degeneration often occurs after posterior lumbar surgery, and back muscle degeneration may be a cause of back pain after surgery. However, the relationship between back muscle degeneration and back pain remains controversial. In the current study, we aimed to classify back muscle degeneration more precisely using MRI and investigate its relationship with back pain after surgery.

\section{Materials and Methods}

\section{Patients}

In our university hospital, we retrospectively evaluated 84 patients, $50-78$ years old (mean \pm standard deviation:
Table 1. Demographic characteristics

\begin{tabular}{lc} 
Characteristic & \\
No. of patients & 84 \\
Sex (male:female) & $44: 40$ \\
\hline Age (yr) & $65.1 \pm 7.8(50-78)$ \\
\hline Symptom duration (mo) & $10.0(4-24)$ \\
\hline Follow-up after surgery (mo) & 12 \\
\hline Diabetes mellitus & 3 \\
\hline Smoker & 4 \\
\hline Pain score before surgery & \\
\hline Low back pain & \\
\hline$\quad$ Visual analogue scale & $5.1 \pm 2.4$ \\
\hline$\quad$ Oswestry disability index & $40 \pm 14$ \\
\hline Leg pain & \\
$\quad$ Visual analogue scale & $8.6 \pm 3.3$ \\
\hline
\end{tabular}

Values are presented as mean (range).

65.1 \pm 7.8 years) who had low back pain and leg pain with lumbar spinal stenosis between January 2005 and April 2013. Patients had low back and leg pain that had persisted for at least 3 months. Patients were diagnosed with one or two levels of lumbar spinal stenosis on X-ray and MRI, myelography, and computed tomography after myelography. Patients who had previously undergone spinal surgery were excluded. We also excluded those with spinal tumors, infections, or acute vertebral fractures of the thoracic and lumbar spine. Informed consent was obtained from each patient. Details of the patients' backgrounds are provided in Table 1.

All patients underwent decompression surgery at the level of the stenosis. One-level or two-level decompression was performed. All patients underwent posterior decompressive surgery via a unilateral approach (left or right side). This approach allows surgeons to perform central and bilateral decompression while only stripping the muscles unilaterally [7].

\section{Radiographic evaluation}

MRI (1.5 tesla) was evaluated before and 12 months after surgery in all patients. CSA of the multifidus muscle on the approach side was measured using a computer-linked digitizer. In patients with one-level stenosis and decompression, we determined the CSA of the multifidus muscle at the level of the corresponding intervertebral disc. In 
patients with two-level stenosis and decompression, we determined the CSA of the multifidus muscle at the center of the mid-vertebra. MRI 12 months after surgery was used to classify muscle degeneration into three types: low intensity in T1-weighted imaging, high intensity in T2weighted imaging (type 1), high intensity in both T1- and T2-weighted imaging (type 2), and low intensity in both T1- and T2-weighted imaging (type 3). The site of evaluation was as mentioned above for the muscle CSA. Evaluation of muscle was performed by three surgeons blinded to the condition. Consensus of at least two of the observers was used to determine the type of degeneration.

\section{Clinical evaluation}

We evaluated the change in low back and leg pain before and 12 months after surgery. To evaluate pain, a visual analogue scale (VAS) score ( 0 , no pain; 10 , worst pain) for low back pain and leg pain, and the Oswestry disability index (ODI) for low back pain were recorded before and 12 months after surgery and compared.

\section{Statistical analyses}

Kruskal-Wallis test was used to compare pain scale data between the three groups, a one-way analysis of varianc with post hoc comparisons was used for follow-up imaging data, and Fisher's test was used for dichotomous or categorical variables. A $p<0.05$ was considered statistically significant.

\section{Results}

Table 1 shows demographic characteristics of the patients before surgery. Fig. 1 shows a representative MRI for each group. MRI revealed muscle degeneration in all patients after surgery (type 1, 6\%; type 2, 82\%; and type 3, 12\%). Type 2 was significantly more prevalent compared with types 1 and $3(p<0.01)$. The average CSA of the multifidus muscle before surgery was not significantly different between the three groups $(p>0.05)$ (Table 2). The average CSA of the multifidus muscle 12 months after surgery was significantly decreased compared with before surgery in all patients $(p<0.01)$ (Table 2$)$. However, there was no
Type 1
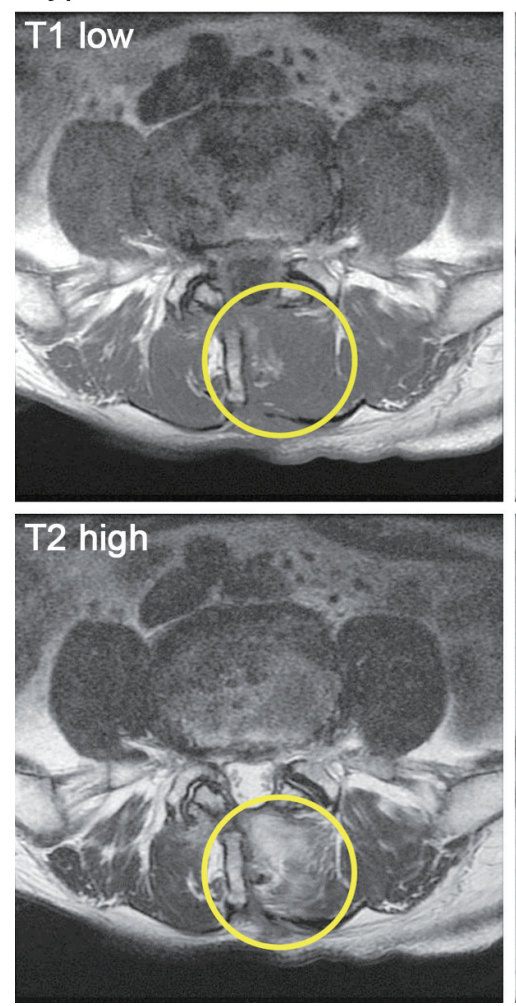

Type 2
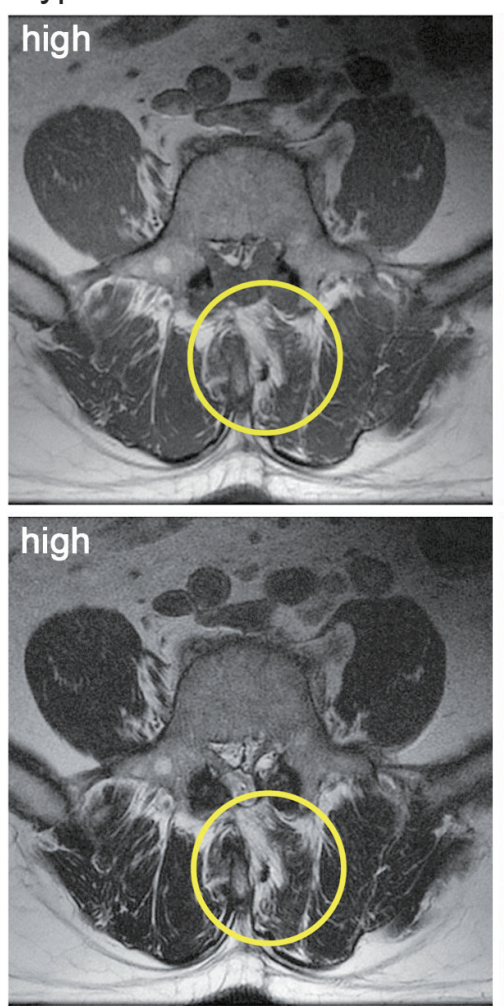

Type 3

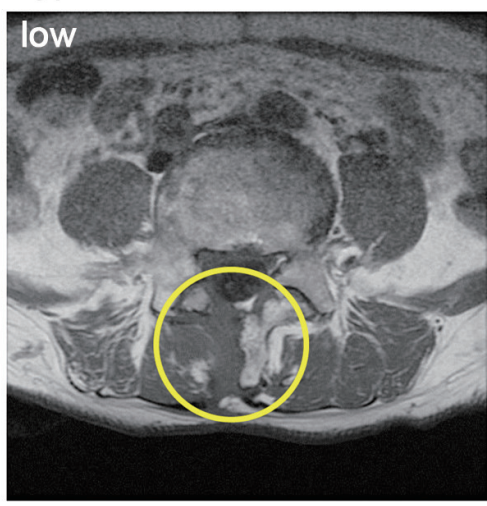

low

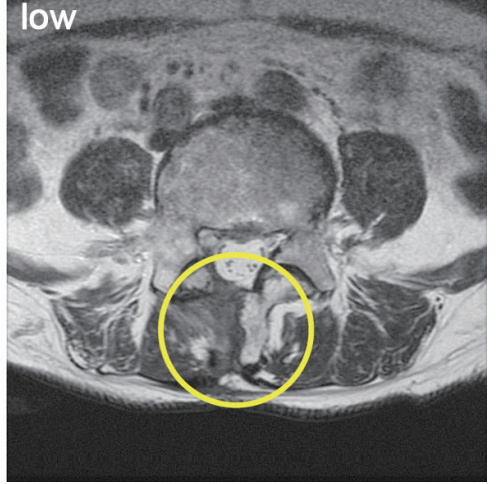

Fig. 1. Representative magnetic resonance imaging for each type of muscle degeneration. 
Table 2. Average of CSA of multifidus muscle before and 12 months after surgery

\begin{tabular}{lcccc} 
Variable & Type 1 & Type 2 & Type 3 & $p$-value \\
No. of patients & 5 & 69 & 10 & - \\
Average of CSA of multifidus muscle before surgery $\left(\mathrm{mm}^{2}\right)$ & $812 \pm 95$ & $843 \pm 105$ & $804 \pm 88$ & 0.22 \\
\hline Average of CSA of multifidus muscle 12 months after surgery $\left(\mathrm{mm}^{2}\right)$ & $610 \pm 90$ & $631 \pm 91$ & $644 \pm 78$ & 0.26 \\
\hline -value (comparison before and after in each group) & 0.032 & 0.01 & -025 \\
\hline
\end{tabular}

CSA, cross-sectional area.

$p<0.05$ was considered statistically significant.

Table 3. Low back pain and leg pain before and 12 months after surgery

\begin{tabular}{lcccc} 
Variable & Type 1 & Type 2 & Type 3 & $p$-value \\
\hline No. of patients & 5 & 69 & 10 & - \\
\hline Low back pain (VAS) before surgery & $5.5 \pm 1.5$ & $6.0 \pm 2.3$ & $5.7 \pm 2.0$ & 0.16 \\
\hline Low back pain (VAS) 12 mo after surgery & $1.8 \pm 0.8$ & $2.0 \pm 0.5$ & $2.2 \pm 0.7$ & 0.23 \\
\hline$p$-value (comparison before and after in each group) & 0.01 & 0.02 & $42 \pm 12$ & - \\
\hline Low back pain (ODI) before surgery & $44 \pm 12$ & $38 \pm 10$ & $26 \pm 6$ & 0.22 \\
\hline Low back pain (ODI) 12 mo after surgery & $24 \pm 8$ & $20 \pm 8$ & 0.04 & -10 \\
\hline$p$-value (comparison before and after in each group) & 0.022 & 0.031 & $8.2 \pm 3.8$ & 0.09 \\
\hline Leg pain (VAS) before surgery & $8.5 \pm 2.3$ & $9.1 \pm 3.3$ & $1.9 \pm 0.7$ & $1.7 \pm 0.8$ \\
\hline Leg pain (VAS) 12 mo after surgery & $2.0 \pm 0.6$ & 0.031 & 0.024 & 0.20 \\
\hline$p$-value (comparison before and after in each group) & 0.043 & - \\
\hline
\end{tabular}

VAS, visual analogue scale; ODI, Oswestry disability index.

A $p<0.05$ was considered statistically significant.

significant difference in the average CSA of the multifidus muscle after surgery between the three groups $(p>0.05)$ (Table 2).

Before surgery there was no significant difference in the low back pain score as evaluated by VAS or ODI, or leg pain score evaluated by VAS between the three groups $(p>0.05)$ (Table 3). Low back pain evaluated by VAS and ODI, and leg pain evaluated by VAS significantly improved after surgery $(p<0.01)$ (Table 3$)$. Low back pain and leg pain were not significantly associated with any of the MRI types of muscle degeneration after surgery $(p>0.05)$ (Table 3).

\section{Discussion}

The current study revealed various pathologies of back muscle degeneration after posterior lumbar surgery. Type 2 (fatty) change was most prevalent, and other patients were classified as having type 3 (scar) or type 1 (inflammation or water-like) changes. Low back pain was not associated with any of the MRI types of muscle degeneration after surgery.

Posterior lumbar surgery is one of the main approaches to deal with lumbar spine disorders. Clinical investigations have noted postoperative injury to and atrophy of the multifidus muscle $[8,9]$ and the extent of atrophy has been significantly associated with postoperative low back pain and functional disability [9]. In the current study, all patients showed muscle degeneration 12 months after surgery. However, low back pain decreased after surgery. In cases of disc herniation or spinal stenosis, we believe that low back pain originates from several structures, including intervertebral discs and spinal nerve roots. Indeed, Toyone et al. [10] reported that in 40 consecutive patients with disc herniation treated by discectomy, all 40 patients were satisfied with the outcome, and suggested that nerve root compression by lumbar disc herniation might be a cause of low back pain. However, patients in the present study still showed slight low back pain 12 months after surgery, and this pain may have originated from degener- 
ated back muscle. The relationship between the extent of back muscle atrophy and postoperative low back pain could not be concluded from the current study.

Presently, type 2 (fatty) muscle degeneration was most prevalent. After posterior surgery in sheep, the main pathological changes noted in the multifidus were the atrophy of muscle bundles, increase in interstices, and decrease in size and number of nuclei [11]. Meanwhile, changes noted on MRI were a decrease in the CSA of the multifidus and infiltration by fibrotic and fatty tissues [11]. Patients with chronic low back pain have a higher percentage of muscle fatty infiltration compared with healthy controls [12]. However, no association between the fat content of the multifidus muscle and pain duration or intensity has been evident [13]. Furthermore, the extent of fatty change in the multifidus muscle is not significantly related with higher ratings of pain intensity [14]. In the current study, low back pain associated with fatty change (type 2) was not significantly different from pain associated with type 1 or 3 atrophy. This finding indicates a lack of association between the fat change in multifidus muscle and postsurgical pain.

Some patients were classified as having type 1 (inflammation or water-like) or type 3 (scar) changes. To our knowledge, there has been no previous report of MRI evaluation of these muscle degeneration changes. Histological findings including muscle degeneration, ballooning of muscle fibers, edema, atrophy, fatty change, and fibrosis and scarring in back muscle from patients who suffered from back pain have been reported [5]. In animal models of posterior lumbar spine surgery, the multifidus muscle has been evaluated histologically and by MRI after surgery [15]. Mainly fatty degeneration occurred. However, necrosis and inflammation and fibrotic change often occur with fatty degeneration [15]. These changes correspond to type 1 and 3 changes seen in the current study.

We wondered whether there is an association between type 1 changes and back pain. We had previously conducted MRI studies of vertebral bone marrow changes in patients with degenerative lumbar disease [16]. Abnormalities associated with decreased signal intensity on T1weighted spin-echo images and increased signal intensity on T2-weighted spin-echo images (Modic type 1) corresponding to the current type 1 change correlated with segmental hypermobility and low back pain [16]. We have also reported that Modic type 1 endplate abnormalities in discogenic pain patients are related to inflammation and axonal growth into the abnormal bone marrow induced by cytokines such as tumor necrosis factor-alpha [17]. However, in the current study, low back pain associated with type 1 change was not significantly different from that associated with type 2 and 3 changes. Further study is needed to clarify the relationship between back pain and the type of muscle degeneration.

The current study has some limitations. First, it is a small-sized retrospective study and the number of patients was limited. Second, we used 1.5 T MRI, which limited resolution. Third, the patient population only included those with lumbar spinal stenosis. As mentioned above, there is the possibility that back pain originated from spinal nerves, other structures, and other muscles, such as the longissimus and the iliocostalis. Fourth, we did not examinethe relationship of fatty infiltration to back muscle and back pain before and after surgery. Fatty infiltration to back muscle has been reported to be crucial factor to back pain [18]. Therefore we could not evaluate pain that was purely associated with postsurgical muscle degeneration.

\section{Conclusions}

Various pathologies of back muscle degeneration were evident after posterior lumbar surgery. Fatty change was most prevalent, and other patients had scarring or inflammatory-like changes. However, no type of back muscle degeneration was correlated with back pain after surgery.

\section{Conflict of Interest}

No potential conflict of interest relevant to this article was reported.

\section{References}

1. Stromqvist B, Fritzell P, Hagg O, Jonss B. Swespine the Swedish Spine Register 2007 reports [Internet]. Falun (Sweden): Swedish Society of Spinal Surgeons; 2007 [cited 2008 Dec 20]. Available from: http:// www.4s.nu/pdf/Report_2007_englishversion.pdf.

2. Kooij A, Meulman JJ. Regression with optimal scaling. In: Meulman JJ, Heiser WJ, editors. SPSS categories 13.0.10. Chicago: SPSS; 2004. pp.107-57.

3. Trief PM, Ploutz-Snyder R, Fredrickson BE. Emotional health predicts pain and function after fusion: 
a prospective multicenter study. Spine (Phila $\mathrm{Pa}$ 1976) 2006;31:823-30.

4. Kawaguchi Y, Matsui H, Tsuji H. Back muscle injury after posterior lumbar spine surgery. Part 2: Histologic and histochemical analyses in humans. Spine (Phila Pa 1976) 1994;19:2598-602.

5. Sihvonen T, Herno A, Paljarvi L, Airaksinen O, Partanen J, Tapaninaho A. Local denervation atrophy of paraspinal muscles in postoperative failed back syndrome. Spine (Phila Pa 1976) 1993;18:575-81.

6. Motosuneya T, Asazuma T, Tsuji T, Watanabe H, Nakayama Y, Nemoto K. Postoperative change of the cross-sectional area of back musculature after 5 surgical procedures as assessed by magnetic resonance imaging. J Spinal Disord Tech 2006;19:318-22.

7. Kinoshita T, Ohki I, Roth KR, Amano K, Moriya H. Results of degenerative spondylolisthesis treated with posterior decompression alone via a new surgical approach. J Neurosurg 2001;95(1 Suppl):11-6.

8. Tsutsumimoto T, Shimogata M, Ohta H, Misawa H. Mini-open versus conventional open posterior lumbar interbody fusion for the treatment of lumbar degenerative spondylolisthesis: comparison of paraspinal muscle damage and slip reduction. Spine (Phila Pa 1976) 2009;34:1923-8.

9. Fan S, Hu Z, Zhao F, Zhao X, Huang Y, Fang X. Multifidus muscle changes and clinical effects of onelevel posterior lumbar interbody fusion: minimally invasive procedure versus conventional open approach. Eur Spine J 2010;19:316-24.

10. Toyone T, Tanaka T, Kato D, Kaneyama R. Low-back pain following surgery for lumbar disc herniation: a prospective study. J Bone Joint Surg Am 2004;86:8936.

11. Liu X, Wang Y, Wu X, et al. Impact of surgical approaches on the lumbar multifidus muscle: an ex- perimental study using sheep as models. J Neurosurg Spine 2010;12:570-6.

12. Kjaer P, Bendix T, Sorensen JS, Korsholm L, LeboeufYde C. Are MRI-defined fat infiltrations in the multifidus muscles associated with low back pain? BMC Med 2007;5:2.

13. Mengiardi B, Schmid MR, Boos N, et al. Fat content of lumbar paraspinal muscles in patients with chronic low back pain and in asymptomatic volunteers: quantification with MR spectroscopy. Radiology 2006;240: 786-92.

14. Kader DF, Wardlaw D, Smith FW. Correlation between the MRI changes in the lumbar multifidus muscles and leg pain. Clin Radiol 2000;55:145-9.

15. Hu ZJ, Fang XQ, Zhou ZJ, Wang JY, Zhao FD, Fan SW. Effect and possible mechanism of musclesplitting approach on multifidus muscle injury and atrophy after posterior lumbar spine surgery. J Bone Joint Surg Am 2013;95:e192.

16. Toyone $\mathrm{T}$, Takahashi $\mathrm{K}$, Kitahara H, Yamagata M, Murakami M, Moriya H. Vertebral bone-marrow changes in degenerative lumbar disc disease: an MRI study of 74 patients with low back pain. J Bone Joint Surg Br 1994;76:757-64.

17. Ohtori S, Inoue G, Ito T, et al. Tumor necrosis factorimmunoreactive cells and PGP 9.5-immunoreactive nerve fibers in vertebral endplates of patients with discogenic low back Pain and Modic Type 1 or Type 2 changes on MRI. Spine (Phila Pa 1976) 2006; 31:1026-31.

18. Hebert JJ, Kjaer P, Fritz JM, Walker BF. The relationship of lumbar multifidus muscle morphology to previous, current, and future low back pain: a 9-year population-based prospective cohort study. Spine (Phila Pa 1976) 2014;39:1417-25. 